\title{
Heredity of Lower Separation Axioms on Function Spaces
}

\author{
Njuguna E. Muturi \\ Department of Mathematics, Egerton University, Egerton, Kenya \\ Email: edward.njuguna@gmail.com
}

Received 23 January 2014; revised 23 February 2014; accepted 28 February 2014

Copyright (C) 2014 by author and Scientific Research Publishing Inc.

This work is licensed under the Creative Commons Attribution International License (CC BY).

http://creativecommons.org/licenses/by/4.0/

(c) (i) Open Access

\section{Abstract}

The set of continuous functions from topological space $Y$ to topological space $Z$ endowed with a topology forms the function space. For $A$ subset of $Y$, the set of continuous functions from the space $A$ to the space $Z$ forms the underlying function space with an induced topology. The function space has properties of topological space dependent on the properties of the space $Z$, such as the $T_{0}, T_{1}, T_{2}$ and $T_{3}$ separation axioms. In this paper, we show that the underlying function space inherits the $T_{0}, T_{1}, T_{2}$ and $T_{3}$ separation axioms from the function space, and that these separation axioms are hereditary on function spaces.

\section{Keywords}

\section{Function Space; Underlying Function Space; Hereditary Properties}

\section{Introduction}

The set of continuous functions from the space $Y$ to the space $Z$ is denoted by $C(Y, Z)$. The set open topology $\tau$ defined on the set $C(Y, Z)$ generated by the sets of the form $F(U, V)=\{f \in C(Y, Z): f(U) \subset V\}$, where the sets $U$ and $V$ ranges over the class $\mathrm{C}$ of compact subsets of $Y$ and $\Omega_{Z}$ class of open subsets of $Z$ respectively, is called the compact open topology. The sets of the form $F(U, V)$ forms subbases for the compact open topology $\tau$ on $C(Y, Z)$ (see [1]). The set open topology $\tau$ defined on the set $C(Y, Z)$ generated by the subbases $S(y, U)=\{f \in C(Y, Z), f(y) \in U\}$ where $y \in Y$ and $U \in \Omega_{Z}$ is called point open topology (see [2]).

Let $A=\bigcap_{i}^{n} U_{i}$ for $\left\{U_{i}: i=1,2,3, \cdots, n\right\}$ family of non-empty open subsets of $Y$. The set $C(A, Z)$ consist 
of continuous functions of the form $f \circ i=\left.f\right|_{A}$ where $i: A \rightarrow Y$ is an inclusion mapping (see [3]).

Let the topological space $Z$ be a $T_{i}$-space for $i=0,1,2,3$, then the function space $C_{\tau}(Y, Z)$ with compact open topology $\tau$ inherits the $T_{i}$-separation axioms for $i=0,1,2,3$ (see [4] and [5]).

Definition 1.1 For $A \subset Y$, the sets of the form

$C(A, Z) \cap S(y, V)=\{f \in C(Y, Z): f(\{y\} \cap A) \in V\}=\left\{f \in C(Y, Z):\left.f\right|_{A}(y) \in V\right\}=S(y, V) \forall y \in A$ as defined in [3], forms the subbases for point open topology on the set $C(A, Z)$.

Definition 1.2 The sets of the form

$C(A, Z) \cap F(U, V)=\{f \in C(Y, Z): f(A \cap U) \subset V\}=\left\{f \in C(Y, Z):\left.f\right|_{A}(U) \subset V\right\}=F(U, V)$ where $U$ is open in $A, U \in C$ and $V \in \Omega_{Z}$, defines the subbases for the set open topology on the set $C(A, Z)$ (see [3]). This topology is referred to as open-open topology (see [6]). If $U$ is compact, then $F(U, V)$ defines the subbases for the compact open topology on the set $C(A, Z)$.

The point open topology and the compact open topology are also open-open topologies. The set $C(A, Z)$ endowed with set open topology $\zeta$ is written as $C_{\zeta}(A, Z)$ and is referred to as the underlying function space of the space $C_{\tau}(Y, Z)$ (see [3]).

Definition 1.3 Let $U_{\circ}$ and $V_{\circ}$ be open subsets of $Y$ and $Z$ respectively. The set $C\left(U_{\circ}, V_{\circ}\right)$ forms the subspace of the function space $C_{\tau}(Y, Z)$ with the induced topology $\varrho$ generated by the subbases $C\left(U_{\circ}, V_{\circ}\right) \cap F(U, V)=\left\{f \in C(Y, Z): f\left(U_{\circ} \cap U\right) \subset\left(V_{\circ} \cap V\right)\right\}=\{f \in C(Y, Z): f(U) \subset V\}=F(U, V)$ (see [7]).

The following lemma and theorem are important for our consideration.

Lemma 1.4 In a regular space, if $F$ is compact, $U$ an open subset of a regular space and $F \subset U$, then for some open set $V, F \subset U$ and $\bar{V} \subset U$.

From the above lemma, the following inference is made. Let $K_{i} \in \mathrm{C}$ where $\mathrm{C}$ is a class of compact subsets of $Y$ and $U_{i} \in \Omega_{Z}$. Then for the space $C_{\tau}(Y, Z)$ with compact open topology $\tau, f\left(K_{i}\right)$ is a compact subset of $U_{i}$. Since $Z$ is a regular space, there exist open sets $V_{i} \in \Omega_{Z}$, such that $f\left(K_{i}\right) \subset V_{i}$ and $\bar{V}_{i} \subset U_{i}$. This implies that $F\left(K_{i}, V_{i}\right) \subset F\left(K_{i}, \overline{V_{i}}\right) \subset F\left(K_{i}, U_{i}\right)$, in which the assertion $\overline{F\left(K_{i}, V_{i}\right)} \subset F\left(K_{i}, \overline{V_{i}}\right)$ can be made (see [5]). [7]).

Theorem 1.5 The function $\sigma: C_{\zeta}(A, Z) \rightarrow C_{\varrho}\left(U_{\circ}, V_{\circ}\right)$ defined by $\sigma\left(\left.f\right|_{A}\right)=f$ is a homeomorphism (see

\section{Lower Separation Axioms on the Underlying Function Space $C_{\zeta}(A, Z)$}

In this section, we show that the underlying function space $C_{\zeta}(A, Z)$ inherits the $T_{i}$-separation axioms for $i=0,1,2,3$ from the space $C_{\tau}(Y, Z)$. Topologies $\tau$ and $\zeta$ are both compact open.

Theorem 2.1 Let the function space $C_{\tau}(Y, Z)$ be a $T_{\circ}$ space. The function space $C_{\zeta}(A, Z)$ for $A \subset Y$ is a $T_{\circ}$ space.

Proof. Let $f, g \in C_{\tau}(Y, Z)$ be distinct maps such that $\forall y \in Y, f(y) \neq g(y)$. Then $\forall y \in A$, $\left.f\right|_{A}(y) \neq\left. g\right|_{A}(y)$. For the open set $S(y, V)$ containing $f$ but not $g$ in $C_{\tau}(Y, Z)$, the open set $C(A, Z) \cap S(y, V)=\{f \in C(Y, Z): f(\{y\} \cap A) \in V\}=\left\{f \in C(Y, Z):\left.f\right|_{A}(y) \in V\right\}=S(y, V)$ in $C_{\zeta}(A, Z)$ contains $\left.f\right|_{A}(y)$ but not $\left.g\right|_{A}(y)$. Therefore the space $C_{\zeta}(A, Z)$ is a $T_{\circ}$ space.

Theorem 2.2 Let the function space $C_{\tau}(Y, Z)$ be a $T_{1}$ space. The function space $C_{\zeta}(A, Z)$ for $A \subset Y$ is a $T_{1}$ space.

Proof. Let $f, g \in C_{\tau}(Y, Z)$ be distinct maps such that $\forall y \in Y, f(y) \neq g(y)$. Then $\forall y \in A$, 
$\left.f\right|_{A}(y) \neq\left. g\right|_{A}(y)$. For the open sets $S(y, V)$ containing $f$ but not $g$ and $S(y, U)$ containing $g$ but not $f$ in $C_{\tau}(Y, Z)$, the open sets

$$
C(A, Z) \cap S(y, V)=\{f \in C(Y, Z): f(\{y\} \cap A) \in V\}=\left\{f \in C(Y, Z):\left.f\right|_{A}(y) \in V\right\}=S(y, V)
$$

and

$$
C(A, Z) \cap S(y, U)=\{g \in C(Y, Z): g(\{y\} \cap A) \in U\}=\left\{g \in C(Y, Z):\left.g\right|_{A}(y) \in U\right\}=S(y, U)
$$

in $C_{\zeta}(A, Z)$ are neighborhoods of $\left.f\right|_{A}(y)$ but not $\left.g\right|_{A}(y)$ and $\left.g\right|_{A}(y)$ but not $\left.f\right|_{A}(y)$ respectively. Therefore the space $C_{\zeta}(A, Z)$ is a $T_{1}$ space.

Theorem 2.3 Let the function space $C_{\tau}(Y, Z)$ be a $T_{2}$ space. The function space $C_{\zeta}(A, Z)$ for $A \subset Y$ is a $T_{2}$ space.

Proof. Let $f, g \in C_{\tau}(Y, Z)$ be distinct maps such that $\forall y \in Y, f(y) \neq g(y)$. Then $\forall y \in A$, $\left.f\right|_{A}(y) \neq\left. g\right|_{A}(y)$. For the disjoint open sets $S(y, V)$ and $S(y, U)$ neighborhoods of $f$ and $g$ respectively in $C_{\tau}(Y, Z)$, the open sets

$$
C(A, Z) \cap S(y, V)=\{f \in C(Y, Z): f(\{y\} \cap A) \in V\}=\left\{f \in C(Y, Z):\left.f\right|_{A}(y) \in V\right\}=S(y, V)
$$

and

$$
C(A, Z) \cap S(y, U)=\{g \in C(Y, Z): g(\{y\} \cap A) \in U\}=\left\{g \in C(Y, Z):\left.g\right|_{A}(y) \in U\right\}=S(y, U)
$$

in $C_{\zeta}(A, Z)$ are disjoint neighborhoods of $\left.f\right|_{A}(y)$ and $\left.g\right|_{A}(y)$ respectively. Therefore the space $C_{\zeta}(A, Z)$ is a $T_{2}$ space.

Theorem 2.4 Let the function space $C_{\tau}(Y, Z)$ be a regular space for a regular space $Z$. The function space $C_{\zeta}(A, Z)$ for $A \subset Y$ is a regular space.

Proof. The space $C_{\tau}(Y, Z)$ is regular for a regular space $Z$ if for the open cover $F\left(K_{i}, U_{i}\right)$ of $f$, there exist open sets $F\left(K_{i}, V_{i}\right) \subset F\left(K_{i}, U_{i}\right)$ neighborhoods of $f$ such that for $g \in C(Y, Z)$ and $g \notin F\left(K_{i}, U_{i}\right)$, $F\left(x, Z \backslash V_{i}\right)$ for some $x \in K_{i}$ is a neighborhood of $g$ which does not intersect $F\left(K_{i}, V_{i}\right)$ and $\overline{F\left(K_{i}, V_{i}\right)} \subset F\left(K_{i}, U_{i}\right)$. For $F\left(K_{i}, V_{i}\right) \subset F\left(K_{i}, U_{i}\right), \quad F\left(K_{i}, V_{i}\right) \cap C(A, Z) \subset F\left(K_{i}, U_{i}\right) \cap C(A, Z)$ implying that $F\left(\cup_{i}, V_{i}\right) \subset F\left(\cup_{i}, U_{i}\right)$, where $\cup_{i}=K_{i} \cap A$. For $g \notin F\left(K_{i}, U_{i}\right)$ we have that $g \notin F\left(\bigcup_{i}, U_{i}\right)$, implying that $\left.g\right|_{A} \notin F\left(\bigcup_{i}, U_{i}\right)$ and for $x \in A,\left.g\right|_{A} \in F\left(x, Z \backslash V_{i}\right)$. Therefore $F\left(x, Z \backslash V_{i}\right)$ is a neighbourhood of $\left.g\right|_{A}$ not intersecting $F\left(\bigcup_{i}, V_{i}\right) . F\left(K_{i}, V_{i}\right) \cap C(A, Z) \subset F\left(K_{i}, \overline{V_{i}}\right) \cap C(A, Z) \subset F\left(K_{i}, U_{i}\right) \cap C(A, Z)$ implies that $F\left(\bigcup_{i}, V_{i}\right) \subset F\left(\bigcup_{i}, \bar{V}_{i}\right) \subset F\left(\bigcup_{i}, U_{i}\right)$. From the assertion $\overline{F\left(K_{i}, V_{i}\right)} \subset F\left(K_{i}, \bar{V}_{i}\right)$ in Lemma 1.4, we have that $\overline{F\left(\bigcup_{i}, V_{i}\right)} \subset F\left(\bigcup_{i}, \bar{V}_{i}\right)$. Therefore $F\left(x, Z \backslash V_{i}\right)$ and $F\left(\cup_{i}, U_{i}\right)$ are two disjoint open sets neighborhoods of $\left.g\right|_{A}$ and $\overline{F\left(\cup_{i}, V_{i}\right)}$ respectively. Hence the set $C(A, Z)$ with the induced topology $\zeta$ is a regular space.

\section{Conclusion}

The underlying function space $C_{\zeta}(A, Z)$ inherits the $T_{i}$-separation axioms for $i=0,1,2,3$ from the function space $C_{\tau}(Y, Z)$. From theorem 1.5, the underlying function space $C_{\zeta}(A, Z)$ is homeomorphic to the subspace $C_{\varrho}\left(\mathrm{U}_{\circ}, \mathrm{V}_{\circ}\right)$ of the function space $C_{\tau}(Y, Z)$. This implies that the subspace $C_{\varrho}\left(\mathrm{U}_{\circ}, \mathrm{V}_{\circ}\right)$ is a $T_{i}$-space for $i=0,1,2,3$, if the function space $C_{\tau}(Y, Z)$ is a $T_{i}$-space for $i=0,1,2,3$. Therefore the $T_{i}$-separation axioms for $i=0,1,2,3$ are hereditary on function spaces. 


\section{References}

[1] Fox, R.H. (1945) On Topologies for Function Spaces. American Mathematical Society, 27, 427-432.

[2] Kelley, J.L. (1955) General Topology. Springer-Verlag, Berlin.

[3] Muturi, N.E., Gichuki, M.N. and Sogomo, K.C. (2013) Topologies on the Underlying Function Space. International Journal of Management, IT and Engineering, 3, 101-113.

[4] Arens, R.F. (1946) A Topology for Spaces of Transformations. The Annals of Mathematics, 2, 480-495. http://dx.doi.org/10.2307/1969087

[5] Willard, S. (1970) General Topology. Addison-Wesley Publishing Company, United States of America.

[6] Kathryn, F.P. (1993) The Open-Open Topology for Function Spaces. International Journal Mathematics and Mathematical Sciences, 16, 111-116. http://dx.doi.org/10.1155/S0161171293000134

[7] Muturi, N.E. (2014) Homeomorphism between the Underlying Function Space and the Subspace of the Function Space. Journal of Advanced Studies in Topology, 1, 57-60. 